\section{Hochrisiko-Weichteilsarkome: Neoadjuvant Anthracyclin/Ifosfamid im Langzeit-Follow-up}

\author{
Patienten mit Hochrisiko-Weichteilsarkomen profitieren von der neoad- \\ juvanten Chemotherapie mit 3 Zyklen Anthrazyklin/Ifosfamid. Das 5-Jahres- \\ Gesamtüberleben (OS) ist nicht schlechter als das nach 5 Zyklen. Nun liegen \\ Daten für das 10-Jahres-OS vor.
}

\begin{abstract}
D ie Standardbehandlung bei lokalisierten Hochrisiko-Weichteilsarkomen umfasst Operation und Strahlentherapie, in der Praxis kommt häufig noch eine zusätzliche adjuvante Chemotherapie dazu. In einer randomisierten Studie war die Überlebenszeit von Patienten mit solchen Sarkomen nach $5 \mathrm{Zy}$ klen Epirubicin/Ifosfamid adjuvant höher als ohne adjuvante Therapie. In einer weiteren Studie mit 328 Hochrisiko-Patienten zeigte sich dann eine Nichtunterlegenheit von 3 präoperativenZyklen Epirubicin $\left(120 \mathrm{mg} / \mathrm{m}^{2}\right)$ plus Ifosfamid $\left(9 \mathrm{~g} / \mathrm{m}^{2}\right)$ im Vergleich zu 5 Zyklen dieses Regimes hinsichtlich des Gesamtüberlebens (OS) nach 5 Jahren. Eine Radiothe-
\end{abstract}

rapie erfolgte prä- oder postoperativ, die Operation fand 3-4 Wochen nach Ende der Chemotherapie statt.

Die Ergebnisse zum 10-Jahres-Follow-up bestätigen die Nichtunterlegenheit der 3 Zyklen. In dieser Zeit waren 58 Patienten der 3-Zyklen- und 65 Patienten in der 5-Zyklen-Gruppe gestorben. Die Rate für das 10-Jahres-OS lag bei 64 bzw. 59\%, das 3-Zyklen-Regime war also dem 5-Zyklen-Regime auch nach median 10 Jahren nicht unterlegen. Die Hazard Ratio von experimenteller versus Kontrollgruppe beim Endpunkt OS betrug 0,92 (95\%-Konfidenzintervall [95\%-KI] 0,68-1,23), beim progressionsfreien Überleben $1,04(0,78-1,38)$.
Patienten mit undifferenzierten pleomorphen Sarkomen erzielten die besten Ansprechraten, die schlechtesten Ansprechraten Patienten mit Leiomyosarkomen. Die Prognosen waren entsprechend besser oder schlechter. Nur wenige Patienten, die bereits 5 Jahre überlebt hatten, entwickelten in den nächsten 5 Jahren ein Rezidiv.

Fazit: Bei Patienten mit HochrisikoWeichteilsarkomen ist eine Behandlung mit 3 Zyklen Anthrazyklin plus Ifosfamid einer Behandlung mit 5 Zyklen auch im 10-Jahres-Follow-up nicht unterlegen. Ob bei bestimmten Subtypen eine entsprechend den histologischen Befunden maßgeschneiderte neoadjuvante Chemotherapie der 3-Zyklen-Therapie überlegen ist, wird derzeit überprüft.

Barbara Kreutzkamp

Gronchi A et al. Short, full-dose adjuvant chemotherapy (CT) in high-risk adult soft tissue sarcomas (STS): long-term follow-up of a randomized clinical trial from the Italian Sarcoma Group and the Spanish Sarcoma Group. Ann Oncol. 2016;27(12):2283-8.

\title{
Myelosuppressive CT: Neutropenie-Risiko vor jedem Zyklus neu ermitteln
}

\section{Zur Risikoabschätzung von Neutropenien unter myelosuppressiver Chemo- therapie gab es bisher nur Prognoseberechnungen vor Beginn der Therapie. Ein neues Modell sagt jetzt das Risiko vor jedem neuen Zyklus voraus.}

$\mathrm{D}$ ie bisher entwickelten Modelle zur Ermittlung des Risikos einer Chemotherapie-induzierten Neutropenie (CIN) bzw. febrilen Neutropenie (FN) beruhen auf Patienten-, Behandlungs- und Krankheitsvariablen, die lediglich zu Beginn der Chemotherapie erhoben werden. Die EORTC favorisiert in ihren aktuellen Empfehlungen allerdings ein Vorgehen, bei dem vor jedem Zyklus das Risiko erneut ermittelt werden sollte. Nun steht erstmals ein solches dynamisches CIN/ FN-Modell für die Risikoabschätzung vor jedem Zyklus zur Verfügung.

Datenbasis für dieses Modell war die MONITOR-G-CSF-Studie. In der unter Praxisbedingungen durchgeführten Untersuchung wurden Therapieverläufe und -ergebnisse von Patienten mit soliden und hämatologischen Tumoren im Stadium 3 oder 4 unter myelosuppressiver Behandlung untersucht. Zur CIN/FN-Prävention kam das G-CSF-Biosimilar EP2006 zum Einsatz, das wirkungsgleich mit dem Referenzprodukt Filgrastim ist.

Wie bei den statischen Modellen werden auch für das dynamische Modell Angaben u.a. zu Anamnese, Risikofaktoren und Therapieintensität benötigt, darüber hinaus Informationen über CIN/FN-Zwischenfälle und den Einsatz von G-CSF-Präparaten in den vorangegangenen Zyklen, die Zykluszahl sowie zentrumsspezifische Charakteristika. Einige der in aufwendigen statistischen Verfahren identifizierten Prädiktoren fanden sich in mehreren der benutzten Einzelmodelle wieder. Neben bekannten Einflussfaktoren wie Patientenalter oder Chemotherapieregime traf dies auch für die Prophylaxe-Intensität zu: Im patienten- sowie im zyklusbezogenen Modell erwies sich eine Unter-Prophylaxe als klinisch unsicher, während eine ÜberProphylaxe möglicherweise nützlich ist - auch bei nur mäßig- oder geringgradig myelosupressiven Regimes.

Fazit: Ein dynamisches CIN/FN-Risikomodell ermöglicht die individuelle Risikoabschätzung bei Patienten unter myelosupressiver Chemotherapie vor jedem Zyklus. Die Ergebnisse beruhen auf einer Biosimilar-Studie unter Real-LifeBedingungen. Die Prophylaxe-Intensität in den vorangegangenen Zyklen war eine zusätzliche, belastbare Prädiktionsvariable.

Barbara Kreutzkamp

Aapro $\mathrm{M}$ et al. Predictive modeling of the outcomes of chemotherapy-induced (febrile) neutropenia prophylaxis with biosimilar filgrastim (MONITOR-GCSF study). Ann Oncol. 2016;27(11):2039-45. 